\title{
Resiliencia e inteligencia emocional en pacientes diagnosticados con trastorno por consumo de sustancias.
}

\author{
Resilience and emotional intelligence in patients diagnosed with substance use disorder. \\ Rosario Andrea Neyra-Elguera ${ }^{1, a}$, Michelle Cano-Dávila ${ }^{1, a}$, Luis Alberto Taype-Huarca ${ }^{2, b}$ \\ RESUMEN
}

El consumo de sustancias psicoactivas se ha relacionado con diversos factores de riesgo y protección en la búsqueda de optimizar la adherencia al tratamiento y prevenir recaídas. Objetivo: Determinar la relación entre resiliencia e inteligencia emocional en pacientes diagnosticados con trastorno por consumo de sustancias. Material y Métodos: Estudio cuantitativo, no experimental, correlacional y transversal. La muestra fue de 43 pacientes, 13 mujeres $(30 \%)$ y 30 varones (70\%), entre 15 y 53 años. Se utilizó la Escala de Resiliencia de Wagnild y Young $(\alpha 0,821)$, y el Inventario Emocional de BarOn ICE $(\alpha$ 0,833). Resultados: Las dimensiones de resiliencia (confianza en sí mismo y perseverancia) tienen influencia significativamente positiva en los factores de inteligencia emocional, i.e., intrapersonal y estado de ánimo general. Conclusiones: En personas con diagnóstico de adicción a sustancias, la capacidad de resiliencia se relaciona positivamente con su habilidad general para afrontar demandas y presiones.

PALABRAS CLAVE: Resiliencia psicológica, inteligencia emocional, adicción a sustancias.

\section{SUMMARY}

The use of psychoactive substances has been related to various risk and protective factors in the search for optimization of treatment adherence and relapse prevention. Objective: To determine the relationship between resilience and emotional intelligence in patients diagnosed with addiction to psychoactive substances. Material and Methods: Quantitative, non-experimental, correlational and cross-sectional study. The sample consisted of 43 patients, 13 women (30\%) and 30 men (70\%) between the ages of 15 and 53. The Wagnild and Young's Resilience Scale $(\alpha$ 0.821) and the BarOn Emotional Quotient Inventory ( $\alpha$ 0.833), were used. Results: The dimensions of resilience (self-reliance and perseverance) have a very significant positive influence on the emotional intelligence factors. i.e,, intrapersonal and general mood. Conclusions: In individuals diagnosed with addiction to psychoactive substances, the resilience capability is positively related to their general ability to face demands and pressures.

KEYWORDS: Psychological resilience, emotional intelligence, substance addiction.

\footnotetext{
Universidad Católica de Santa María. Arequipa, Perú.

Escuela Profesional de Psicología, Universidad Católica de Santa María. Arequipa, Perú.

a Licenciada en Psicología

b Psicólogo, Docente.
} 
Resiliencia e inteligencia emocional en pacientes diagnosticados con trastorno por consumo de sustancias.

\section{INTRODUCCIÓN}

La adicción, la forma más severa de trastorno por consumo de sustancias, es un trastorno cerebral crónico moldeado por fuertes factores biosociales que tiene consecuencias devastadoras para los individuos y para la sociedad (1)

El DSM-5 especifica que los síntomas del trastorno por consumo de sustancias puede incluir tolerancia; abstinencia; pérdida de control; esfuerzos fallidos por reducir o dejar el consumo; una gran cantidad de tiempo empeñado en buscar, usar o recuperarse del uso de sustancias; deterioro en áreas específicas de la vida del sujeto; y un continuo uso a pesar de las consecuencias negativas. El diagnóstico se hace cuando la persona tiene 2 o más síntomas de 11 a lo largo de un periodo de tiempo de 12 meses (2).

La información que se muestra en el Informe Mundial sobre las Drogas 2018 muestra la trascendencia y complejidad de los mercados mundiales de drogas, las consecuencias que las drogas traen para la salud son considerables, en África 5000 personas consumen drogas por medio de inyectables, siendo la principal causa de VIH en donde un $44 \%$ de dicha población se ve afectada, así también un 95\% de estos consumidores sufren de Hepatitis C producidas por agujas infectadas, en Asia el $24 \%$ de los consumidores por inyectables viven con VIH, entre los años 2016 y 2017 en América del Norte los casos de sobredosis aumentaron un $29,7 \%$ siendo la mayoría causadas por opioides ; las muertes son otras de las consecuencias del consumo, en América del Norte la tasa de mortalidad de 16 estados se vieron afectados por el aumento de muertes causadas por sobredosis en un $34,5 \%(3,4)$.

En el Perú, la información sobre la trascendencia del uso de drogas y sus efectos económicos, sociales y sanitarios, muestran a esta situación problemática como relevante para poner a trabajar las políticas públicas e intervenciones que minimicen la exposición e impacto de factores de riesgo y refuercen los factores protectores en los individuos (5).

La prevalencia de vida es un indicador general que refleja la tasa de personas que han usado una sustancia al menos una vez en la vida. Según CEDRO, en una muestra aplicada a 13 ciudades de Perú, la prevalencia es mayor para las drogas legales como el alcohol (79\%) y el tabaco (53\%). La prevalencia de vida de las drogas ilegales es mayor para la marihuana $(8 \%)$, el PBC (2\%) y el clorhidrato de cocaína (2\%), en todos los casos el consumo se da en mayor proporción por hombres que por mujeres (6).

Al trabajar con pacientes adictos, el equipo de intervención busca la rehabilitación y prevención de recaídas, con ese fin, es necesario fortalecer las herramientas de trabajo terapéutico al conocer el funcionamiento, la influencia o relación de los factores protectores como la capacidad de recuperación, el funcionamiento familiar, la adhesión al tratamiento, la resiliencia o la inteligencia emocional entre otros.

La resiliencia es la capacidad de hacer las cosas bien pese a las condiciones de vida adversas, a las frustraciones, superarlas y salir de ellas fortalecido o incluso transformado (7).

La resiliencia se refiere al proceso dinámico que abarca la adaptación positiva dentro de un contexto de adversidad significativa (8). Es el resultado de la combinación o interacción entre los atributos del individuo (internos) y los propios de su ambiente familiar, social y cultural (externos) que lo posibilitan para superar el riesgo y la adversidad de forma constructiva (9).

Si se fortalece la resiliencia en las personas, esto supondría un factor protector ante el uso de drogas, debido a que con ella, la persona puede solucionar situaciones riesgosas, incómodas, evitando desarrollar conductas problemáticas como el consumo de alcohol o drogas (10). Algunos estudios han tratado de conocer la influencia de la resiliencia en pacientes con este tipo de diagnóstico como factor de protección (11), también se han desarrollado programas de prevención enfocados en resiliencia, frente al consumo de sustancias psicoactivas (12). La inteligencia emocional tiene que ver con la capacidad de reconocer lo que sentimos nosotros y otras personas, y encontrar formas de lidiar con esas emociones (13). Además, supone la capacidad de discernir adecuadamente; determinar y manifestar emoción; acceder y/o producir sentimientos; comprender y conocer las emociones; y por último la habilidad de moderar las emociones para fomentar el desarrollo emocional e intelectual (14).

Estudios han enfocado la importancia de la inteligencia emocional sobre la recuperación de pacientes con trastorno por consumo de sustancias, actuando como variable moduladora del comportamiento final, también puede actuar como una medida preventiva de eficacia en los programas de prevención de las adicciones (15). Existe la 
necesidad de implementar programas que incluyan en sus protocolos de abordaje la inteligencia emocional y la resiliencia en la rehabilitación de adictos, siendo ambos de gran interés para una intervención efectiva. En este estudio se busca establecer la correlación entre resiliencia e inteligencia emocional en pacientes diagnosticados con trastorno por consumo de sustancias.

\section{MATERIAL Y MÉTODOS}

El presente es un estudio cuantitativo, no experimental de alcance correlacional y tipo transversal; de muestreo no probabilístico, intencional (16).

Participaron 43 sujetos diagnosticados con trastorno por consumo de sustancias; sus edades oscilaban entre los 15 y 53 años, 30 del género masculino y 13 del femenino (tabla 1). Todos los evaluados tenían al menos grado de instrucción primaria completa, y se encontraban hospitalizados o asistían a consulta externa del Complejo Hospitalario Moisés Heresi, de la ciudad de Arequipa, Perú. Por otro lado los pacientes no presentaban deterioro cognitivo que les limite a no poder contestar los cuestionarios, tampoco presentaban comorbilidad psicótica.

Se solicitaron los permisos correspondientes en el establecimiento salud para poder aplicar los instrumentos de evaluación. Mediante oficios y entrevistas se explicó los objetivos, importancia y procedimientos de la investigación.

Luego de la aceptación de las autoridades se coordinaron las fechas y los tiempos más convenientes para realizar la administración de los instrumentos.

La evaluación de cada participante se llevó a cabo en 1 sesión con una duración aproximada de 35 minutos, entre los meses de mayo a junio del 2019, respectivamente. Se explicó a los participantes los alcances del estudio y se recalcó que su participación

Tabla 1. Descripción de la muestra

\begin{tabular}{lcc}
\hline & Género & \\
\hline & $\mathrm{F}$ & $\%$ \\
Femenino & 13 & 30.23 \\
Masculino & 30 & 69.77 \\
Total & 43 & 100 \\
\hline
\end{tabular}

era de carácter anónima y voluntaria. Todos los participantes firmaron un consentimiento informado o un asentimiento en el caso de los menores de edad.

\section{Instrumentos utilizados}

\section{Escala de Resiliencia de Wagnildy Young}

Fue desarrollada en Estados Unidos; traducida y adaptada en el Perú por Novella en el 2002 (17), es de administración individual y colectiva, con una duración aproximada de 25 a 30 minutos, la aplicación es para adolescentes y adultos. Evalúa las dimensiones de la resiliencia: Ecuanimidad, Sentirse bien solo, Confianza en sí mismo, Perseverancia y Satisfacción. Considera también una Escala total. Los estudios citados por Wagnild y Young dieron confiabilidades de 0,85 en una muestra de cuidadores de enfermos de Alzheimer; 0,86 y 0,85 en dos muestras de estudiantes femeninas graduadas; 0,90 en madres primerizas post parto; y 0.76 en residentes de alojamientos públicos. Además, con el método test retest la confiabilidad fue evaluada por los autores en un estudio longitudinal en mujeres embarazadas antes y después del parto, obteniéndose correlaciones de 0,67 a 0,84 , los cuales son respetables. La prueba piloto realizada por Novella en el 2002 tuvo una confiabilidad calculada por el método de la consistencia interna con el coeficiente alfa de Cronbach de 0.89 (18). En esta investigación este instrumento obtuvo un Alfa de Cronbach de 0.821.

\section{Inventario de Inteligencia Emocional de Reuven Bar-On}

El Inventario de Inteligencia Emocional de Reuven Bar-On, fue hecha en Toronto Canadá, fue adaptada en Perú por Nelly Ugarriza Chávez (19). El Inventario de Coeficiente de Inteligencia Emocional de Bar-On Abreviado para Jóvenes y Adultos consta de 60 ítems, estos evalúan 5 componentes y 14 subcomponentes. Este inventario cuenta con una adaptación desarrollada en Lima, hecha por Ugarriza en el 2003, la validación de protocolo del test abreviado fue hecha en la muestra de la investigación de Raúl Andrés Alejos Vera y Sonia Milagros Cambero Aguilar, en el año 2017, es de administración individual y colectiva tipo cuadernillo y digital, su duración aproximada es de 15 a 20 minutos, la aplicación es para adolescentes y adultos con nivel lector de sexto grado de primaria, este test puede ser utilizado en el área educacional, clínico, médico, laboral y en la investigación. En esta investigación se halló un Alfa de Cronbach total de 0,833 . 
Se evaluaron los puntajes de ambos instrumentos, los datos fueron tabulados con el programa Microsoft Excel, para luego aplicar la estadística respectiva en el Sofware IBM SPSS 21, así mismo se realizó la comprobación de la estadística con el coeficiente de correlación Chi cuadrado y Spearman.

El presente estudio fue revisado y aprobado por el Comité de Ética Institucional de Investigación de la Universidad Católica de Santa María (UCSM).

\section{RESULTADOS}

Las correlaciones entre resiliencia e inteligencia emocional se pueden apreciar en la tabla 2. La correlación entre las variables es significativa y en la dirección esperada.

Como se aprecia en el diagrama de dispersión del gráfico 1 , los niveles de resiliencia se relacionan directamente con los niveles de inteligencia emocional. Algunos puntos no siguen un patrón exacto, pero la tendencia global es clara desde la izquierda inferior a la derecha superior del gráfico.
Sobre la relación entre los niveles de resiliencia y los niveles de inteligencia emocional, en la tabla 3 podemos ver que los evaluados con niveles medios de resiliencia, se ubican en su mayoría en niveles altos de inteligencia emocional $(7,7 \%)$, mientras que los que presentan niveles medios se ubican a su vez en niveles medios de inteligencia emocional (84,6\%), asimismo aquellos con niveles bajos de resiliencia se ubican en niveles bajos de inteligencia emocional (75\%).

En el análisis de las dimensiones de resiliencia y componentes de inteligencia emocional que apreciamos en la tabla 4, se observan relaciones estadísticamente significativas entre las dimensiones de sentirse bien solo, confianza en sí mismo y perseverancia con los componentes intrapersonal, adaptabilidad y estado de ánimo general; por otro lado, existe una relación significativa entre la dimensión de ecuanimidad y el componente intrapersonal; cabe mencionar que la dimensión de sentirse bien solo de la resiliencia se asocia significativamente con todos los componentes de la inteligencia emocional a excepción del manejo del estrés.

Tabla 2. Resiliencia e inteligencia emocional

\begin{tabular}{lcc}
\hline & Resiliencia & Inteligencia emocional \\
\hline Resiliencia & 1 & $0,708^{* *}$ \\
Inteligencia emocional & 1 \\
\hline$* *$ p $<0,01$ & \\
Nota: Se utilizó el coeficiente de correlación de Spearman.
\end{tabular}

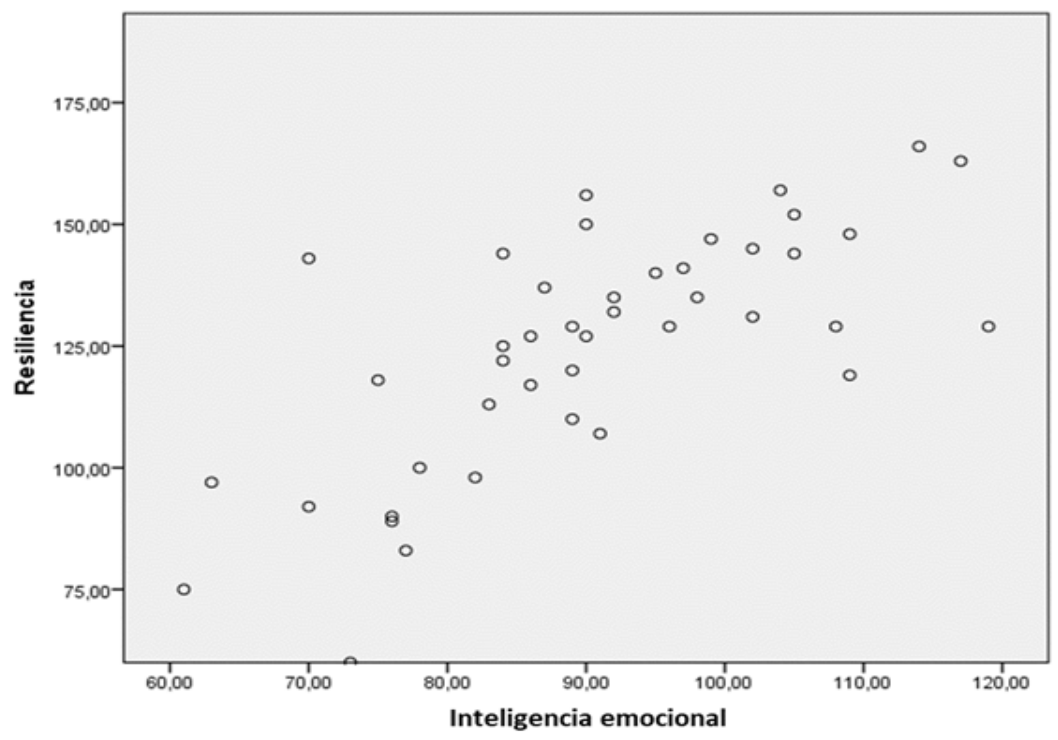

Gráfico 1. Nube de puntos de resiliencia e inteligencia emocional 
Tabla 3. Niveles de resiliencia e inteligencia emocional

\begin{tabular}{|c|c|c|c|c|c|c|c|}
\hline & \multicolumn{7}{|c|}{ Cociente Emocional General } \\
\hline & \multicolumn{2}{|c|}{ Alto } & \multicolumn{2}{|c|}{ Medio } & \multicolumn{2}{|c|}{ Bajo } & \\
\hline & Recuento & $\%$ & Recuento & $\%$ & Recuento & $\%$ & \\
\hline \multirow{3}{*}{$\begin{array}{l}\text { Global } \\
\text { Resiliencia }\end{array}$} & Alto & 1 & 7,1 & 11 & 78,6 & 2 & 14,3 \\
\hline & Medio & 1 & 7,7 & 11 & 84,6 & 1 & 7,7 \\
\hline & Bajo & 0 & 0,0 & 4 & 25,0 & 12 & 75,0 \\
\hline Total & & 2 & 4,7 & 26 & 60,5 & 15 & 34,9 \\
\hline
\end{tabular}

Tabla 4. Dimensiones de resiliencia y componentes de inteligencia emocional

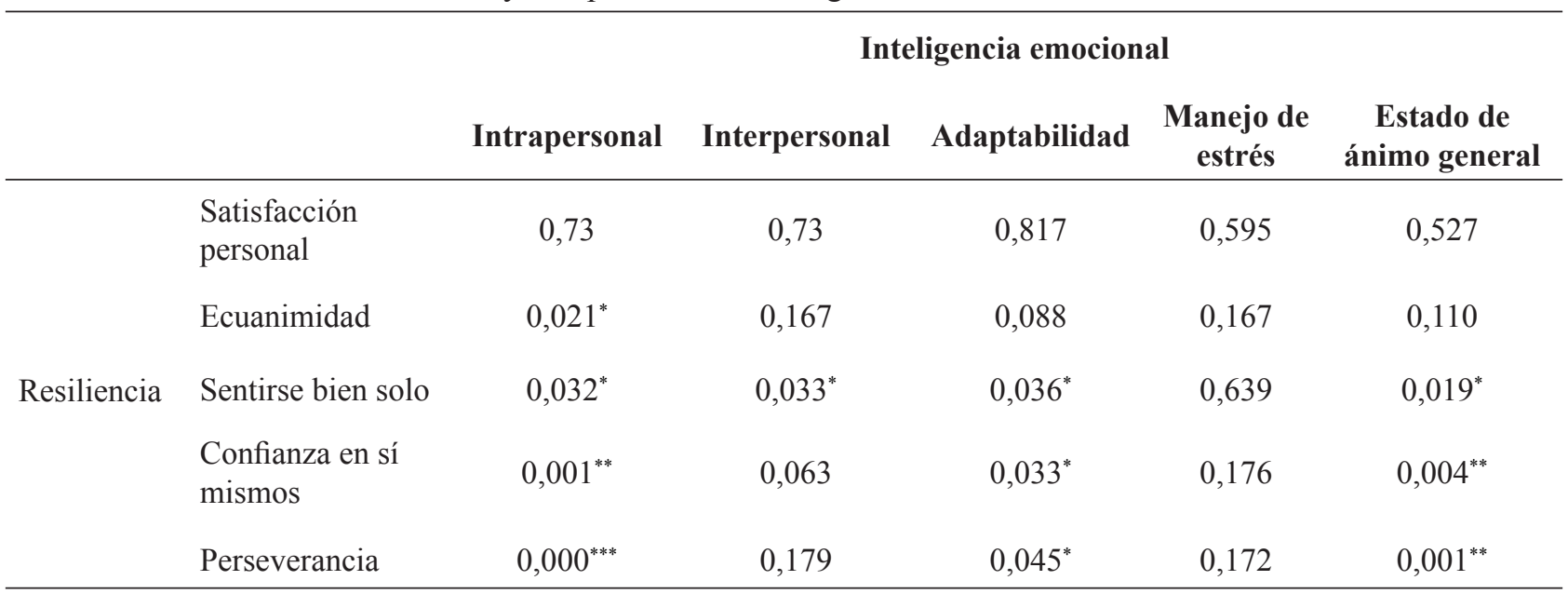

$* \mathrm{p}<0.05 ; * * \mathrm{p}<0.01 ; * * * \mathrm{p}<0.001$.

Nota: Se utilizó la prueba estadística Chi Cuadrado.

\section{DISCUSIÓN}

El trabajo con pacientes con adicciones, busca la rehabilitación y la prevención de recaídas, con ese fin, es necesario fortalecer las herramientas terapéuticas, estableciendo factores protectores. Algunas investigaciones reportan que los sujetos que posean mayor resiliencia podrían rehabilitarse de una manera más rápida, esto permitiría al paciente sobreponerse a una posible futura recaída, actuando como un elemento personal protector $(10,20)$. Así también la inteligencia emocional, permitiría al paciente gestionar sus impulsos emocionales desbordantes, que con frecuencia preceden a un consumo excesivo de sustancias psicoactivas y llevan a posibles recaídas $(15,21)$.

Los resultados obtenidos en este estudio demuestran que existe una relación positiva y altamente significativa entre las variables de resiliencia e inteligencia emocional en pacientes adictos. Estos resultados son similares a algunas investigaciones (22) y parcialmente discrepantes con otros (23).

La resistencia al consumo de drogas depende principalmente de la interacción entre los factores de riesgo y los factores protectores en las diferentes etapas de la vida. Debido a esto las intervenciones destinadas a mejorar la resiliencia que den lugar a un mayor apoyo social podrían ser particularmente eficaces para ayudar a las personas a hacer frente a los factores de riesgo y prevenir la aparición de problemas de consumo de drogas y las recaídas. Sin embargo, como también sucede en nuestros hallazgos la resiliencia no es permanente sino fluctuante; cambia el equilibrio entre los factores de riesgo y los de protección para que los efectos de los factores de protección superen a los de los factores de riesgo (24). Por otro lado, en la dependencia de sustancias algunas características juegan roles cruciales: rasgos de 
Resiliencia e inteligencia emocional en pacientes diagnosticados con trastorno por consumo de sustancias.

personalidad, relaciones sociales, actitudes y valores, así como elementos de inteligencia emocional, la autoestima y relaciones interpersonales. Estudios previos hallaron que la inteligencia emocional tiene una relación significativa con la personalidad inestable en los abusadores de sustancias (25).

Los resultados de este estudio coinciden con lo reportado por Molero-Jurado et al., (22), se halló que los no usuarios de alcohol y tabaco tuvieron puntajes significativamente más altos en inteligencia emocional y resiliencia, a diferencia del grupo de consumidores quienes presentaban puntajes bajos.

Por otro lado existen puntos parcialmente discrepantes en nuestros resultados con los de Martínez quien investigó la resiliencia e inteligencia emocional como predictores de la disminución y cese del consumo de cocaína con adultos politoxicómanos en España. En este estudio se encontraron dos resultados interesantes (a) que las personas más resilientes mostraban consumos más bajos de cocaína y (b) los pacientes consumidores presentaban mayor inteligencia emocional(23).

Nuestros resultados indican que los participantes tienen niveles bajos y medios de resiliencia y en su mayoría presentan niveles medios de inteligencia emocional, esto nos dice que la mayoría de ellos no tienen un perfil bajo en este componente, es decir tienen habilidades emocionales que les permiten afrontar demandas y situaciones de estrés. Las diferencias con el estudio de Martínez pueden deberse a la cantidad de muestra y a las características del instrumento usado para medir inteligencia emocional (23).

Por otro lado, Yang, Zhou, Cao, Xia y An evaluaron; por medio de un estudio transversal realizado en China, a 298 pacientes que presentaban trastornos por consumo de sustancias psicoactivas; la relación entre el autocontrol, la autoeficacia, la autoestima y la resiliencia, se encontró que las variables en mención podían predecir la autoeficacia en los pacientes. Esto nos indica que el aumento del autocontrol, resiliencia y autoestima pueden mejorar o incrementar la autoeficacia en pacientes que presentaban trastornos por consumo de sustancias psicoactivas (26).

Dentro de las limitaciones del presente estudio, podemos mencionar que en el caso de los pacientes hospitalizados y semi residenciales, realizaban varias actividades previamente programadas en el día, lo cual dificultó su evaluación. Por otro lado, no se pudo evaluar a los pacientes nuevos debido a que su tiempo en cada consultorio era muy reducido y aún no contaban con el diagnóstico de adicción. Estas limitaciones redujeron la muestra.

A través de esta investigación, se ha logrado visibilizar y dar énfasis a dos aspectos posiblemente útiles en la rehabilitación de pacientes con adicciones. Los resultados, no difieren en gran medida de otros reportes. Es fundamental proponer nuevos estudios que incluyan variables adicionales como personalidad, capacidad de recuperación, el funcionamiento familiar, la adhesión al tratamiento y otras variables psicosociales que puedan llevar a la elaboración de programas de utilidad para los equipos de intervención.

Financiamiento: El presente trabajo fue autofinanciado. Conflictos de interés: Los autores de este manuscrito declaramos no tener ningún conflicto de interés. El estudio ha sido presentado previamente como parte de una tesis de grado de las dos primeras autoras siendo el último autor el asesor senior.

\section{Correspondencia:}

Rosario Andrea Neyra-Elguera

Universidad Católica de Santa María

Urbanización San José s/n Umacollo, Arequipa - Perú. Correo electrónico: rsr.neyra@gmail.com

\section{REFERENCIAS BIBLIOGRÁFICAS}

1. Volkow N, Boyle M. Neuroscience of Addiction: Relevance to Prevention and Treatment. American Journal of Psychiatry. 2018;175(8):729-740.

2. American Psychiatric Association. Diagnostic and statistical manual of mental disorders. 5th ed. Arlington: American Psychiatric Association; 2014.

3. Oficina de las Naciones Unidas contra la Droga y el Delito. Informe de la Junta Internacional de Fiscalización de Estupefacientes correspondiente a 2016. Nueva York: Organización de las Naciones Unidas; 2017.

4. The United Nations Office on Drugs and Crime. World Drug Report. Vienna: United Nations Publications; 2018.

5. Moreno A, Masías C, Delgado I, Islas J, Ochoa M, Guzmán V, et al. Estrategia Nacional de Lucha Contra las Drogas 2017-2021. Lima: Comisión Nacional para el Desarrollo y Vida sin Drogas; 2017.

6. Centro de Información y Educación para Prevención del Abuso de Drogas .El problema de las drogas en el Perú. Lima: Centro de Información y Educación para Prevención del Abuso de Drogas; 2018. 
7. Wagnild GM, Young HM. Development and psychometric evaluation of the Resilience Scale. J Nurs Meas. 1993;1(2):165-178.

8. Luthar SS, Cicchetti D, Becker B. The construct of resilience: a critical evaluation and guidelines for future work. Child development. 2000;71(3):543562.

9. González-Arratia NI, Valdez JL, Oudhof H, González S. Resiliencia y factores protectores en menores infractores y en situación de calle. Psicología y Salud. 2012;22:49-62.

10. Becoña E. Resiliencia y consumo de drogas: una revisión. Adicciones. 2007;19(1):89-101.

11. Moral-Jiménez M. Personalidad, resiliencia y otros factores psicosociales asociados al consumo desustancias psicoactivasenlaadolescencia:propuesta etiológica. Revista Española de Drogodependencias. 2007;32(3):250-291.

12. Halmi A, Golik-Gruber V. The prevention and reduction of the consumption of alcohol and any other drugs among a high-risk group of youths through improving family resilience. Alcoholism. 2002;38:4155.

13. Martins A, Ramalho N, Morin E. A comprehensive meta-analysis of the relationship between Emotional Intelligence and health. Personality and Individual Differences. 2010;49(6):554-564. Doi: https://doi. org/10.1016/j.paid.2010.05.029

14. Salovery P, Sluyter D. Emotional Development and Emotional Intelligence: Educational implications. New York: Basic Books; 1997.

15. García JA, del-Castillo-López ÁG, Gázquez-Pertusa M, Marzo JC. La inteligencia emocional como estrategia de prevención de las adicciones. Salud y Drogas. 2013;13(2):89-97.

16. Hernández-Sampieri R, Mendoza CP. Metodología de la investigación. Ciudad de México: Mc Graw Hill Education; 2018.

17. Salgado C. Medición de la resiliencia en adolescentes y adultos. Temática Psicológica. 2012;8(1):21-27.

18. Novella A. Incremento de la resiliencia luego de la aplicación de un programa de psicoterapia breve en madres adolescentes. Tesis de Maestría. Lima, Perú: Universidad Nacional Mayor de San Marcos; 2002.
19. Ugarriza N. La evaluación de la inteligencia emocional a través del inventario de BarOn (I-CE) en una muestra de Lima Metropolitana. Lima: Ediciones Libro Amigo; 2003.

20. García J, García M, Rivera S. Potencial resiliente en familias con adolescentes que consumen y no consumen alcohol. Acta Colombiana de Psicología 2015;18(2):163-172. Disponible en: http://editorial. ucatolica.edu.co/ojsucatolica/revistas_ucatolica/ index.php/acta-colombiana-psicologia/article/ view/123 20/04/2020

21. Limonero JT, Fernandez-Castro J, Gomez-Romero MJ, Tomas-Sabado J. Influencia de la inteligencia emocional percibida y la impulsividad en el abuso de cannabis en jóvenes. Ansiedad y Estrés. 2013;19(23):223-234.

22. Molero-Jurado MM, Pérez-Fuentes MC, BarragánMartín AB, del Pino-Salvador RM, Gázquez-Linares JJ. Analysis of the relationship between emotional intelligence, resilience, and family functioning in adolescents' sustainable use of alcohol and tobacco. Sustainability. 2019;11:29-54.

23. Martínez A. Importancia de la Resiliencia e Inteligencia Emocional en el consumo de cocaína. Tesis de Doctorado. Albacete: Universidad de Castilla-La Mancha; 2015.

24. El Rawas R, Amaral IM, Hofer A. Social interaction reward: A resilience approach to overcome vulnerability to drugs of abuse. European Neuropsychopharmacology. 2020;37:12-28.

25. Haj Hosseini F, Mehdizadeh Zare Anari A. The Correlation between Emotional Intelligence and Instable Personality in Substance Abusers. Addict Health. 2011;3(3-4):130-136. doi: 10.1016/S09249338(12)74234-7

26. Yang C, Zhou Y, Cao Q, Xia M, An J. The relationship between self-control and self-efficacy among patients with substance use disorders: Resilience and self-esteem as mediators. Frontiers in Psychiatry. 2019;10:388. doi: 10.3389/fpsyt.2019.00388

Recibido: 03/06/2020

Aceptado: 14/12/2020 